\title{
OCCUPATIONAL DERMATITIS IN DENTISTS: SUSCEPTIBILITY TO PROCAIN
}

\author{
C. GUY LANE, M.D.
}

Assistant Dermatologist, Massachusetts General Hospital; Assistant Physician, Department of Dermatology, Boston Dispensary

BOSTON

Occupational dermatitis in dentists has usually been laid at the door of some one of their antiseptics, or soaps, or in an indefinite way referred to some one of the many substances which they handle. Frequently the patient, a dentist, is informed that sooner or later experience will show the particular agent which is causing his condition. An examination of the textbooks and literature does not reveal, except in one instance, any evidence of a dermatitis associated with local anesthetics. Mook, ${ }^{1}$ in a recent paper, has reported the case of a dentist with susceptibility to apothesin, and in reporting this case states that procain gave much the same reaction as apothesin when skin tests were applied. The dermatitis in this case, however, cleared up when the use of apothesin was stopped. The attention of the profession has apparently not been called to the fact that procain may be an etiologic factor in some of these cases. It is the factor, without doubt, in the three cases which I have to report. These dentists, who have practiced for a number of years, who have used procain over a period of months, have had an irritated condition of the hands, which appears to be due to their individual susceptibility to procain. This dermatitis did not develop immediately when they began to use the drug, but appeared after it had been employed by them as a local anesthetic over a period of months.. This fact may be interpreted as an indication that the condition was fundamentally an acquired susceptibility rather than a real idiosyncrasy-a susceptibility of unexplained or idiopathic origin.

The fact that procain can be added to the list of substances which are the cause of cutaneous manifestations in susceptible persons warrants a few statements about the drug. Procain was introduced by Einhorn, and was clinically tested and exhaustively written about by Braun in 1905. It was most widely used among dentists, and also for local anesthesia in surgery. It was introduced into this country about 1912, and is apparently the local anesthetic most widely used among

1. Mook, W. H.: Skin Reactions to Apothesin and Quinin in Susceptible Persons, Arch. Dermat. \& Syph. 1:651 (June) 1920. 
dentists, and also for local anesthesia in surgery. It has numerous advantages over cocain. It is six or seven times less toxic; it will keep for a long time without deterioration; its action is increased and prolonged by combining it with epinephrin; it does not cause the least irritation on injection; it is soluble in its own weight of water. Le Brocq makes the statement that it is equal to cocain in its anesthetic properties. Gwathmey is responsible for the statement that after absorption the general effect on the system is scarcely perceptible, neither the circulation nor the respiratory system being affected. There is no mydriasis, no disturbance of accommodation, and no increase of intra-ocular pressure. It is neutral in reaction, and its solutions are precipitated by alkaloids and alkaline carbonates, although it is not affected by sodium bicarbonate. It is apparently a much more satisfactory drug to use than cocain.

The three dentists whom I have seen recently because of a dermatitis of the hands have been in practice for many years, and have been using procain for some months. The eruption on their hands has appeared since they began to use this drug. Since discontinuing its use, or wearing gloves, the irritation has disappeared and their hands have become normal. Furthermore, the evidence of skin tests has been in agreement with the clinical evidence.

\section{REPQRT OF CASES}

CASE 1.-Dr. A. has practiced dentistry for twenty-three years. He has used procain occasionally for one year, and exclusively for two years.

History.-In May, 1920, itching began between the fourth and fifth fingers on the right hand, which gradually extended to other fingers; the fingers became red and fissured, and there was some oozing. Itching had been most intense at times.

Examination.-Examination in July showed considerable scaling, with fissuring of both palms and about the fingers. There were circinate, almost serpiginous areas with numerous scales, considerable redness, and a suggestion of vesiculation. There was much redness between several of the fingers, and there was a deep fissure between the fourth and fifth fingers on the right hand, and a fairly deep fissure at the base of the fourth finger on the palmar surface, in addition to other less active fissures. The condition of the right hand was much worse than that of the left hand, especially on the ulnar side. The palms, at this visit, gave the impression of an epidermophyton infection. Examination of the scales was negative, but the clinical appearance warranted the tse of halfstrength Whitfield's ointment. Examination of the scales at the end of twentyfour hours in potassium hydrate solution revealed no mycelia, but there were numerous highly refractile bodies suggesting spores.

Course and Treatment.--In two weeks there was distinct improvement, both objectively and subjectively. The itching became less, and the fissures healed, leaving only slightly scaly and red areas between the fingers and the sites of former open fissures. The skin became more normal. An area of vesiculation and redness appeared about the nose, right cheek and lip, which at this time 
was thought to be caused by the irritation which resulted from the transference of some of Whitfield's ointment to the face.

At the end of two weeks more, new areas had appeared on the hands, areas which were more vesicular and grouped, with recurrence of fissures and burning and itching, especially on the middle and forefingers of the left hand. At this time inquiry was made into the chemicals which the patient was using, particularly the ones which he had begun to use most recently. At this time procain was suggested as a possible cause, and he was advised to use rubber gloves during his work, and to alternate between a weak Whitfield's and Lassar's paste. His hands became better, the fissures closed, and there remained numerous areas of dry, scaly skin, with few fissures which were dry. There was no redness and no vesicles, and the itching had gradually disappeared.

Skin Tests.-Six weeks after his first visit skin tests were made on his forearm with the solution of procain which he customarily used. It contained procain, 2 per cent., in Ringer's solution, with a slight amount of chloretone as preservative. The tests were performed, using this solution, with and without suprarenal extract, and using an old solution made up ten days or two weeks previously. In twenty-four hours there was intense redness and itching at the points of inoculation with all procain solutions. The old solution gave the greatest reaction, showing a rather intense red area, $1 \mathrm{~cm}$. in diameter, elevated, with severe itching. Controls were negative.

At this time he went on his vacation and on his return in three weeks reported that his hands were normal while away, even though subjected to fairly hard usage. They were just as well as ever on his return to work. $\mathrm{He}$ has continued using procain solution, but has worn gloves when using the solution. In spite of this fact, he has one or two small fissures on his hands, and there is occasionally more or less itching. He has had slight irritation and redness about the right corner of his mouth, and recalls squirting some of the procain solution on his face several days before. The previous irritation on his face probably resulted from a similar accident. On his forearm there are still redness and slight thickening of the skin at the site of previous inoculations the point of application of the old procain solution showing as a distinctly purplish area about $1 \mathrm{~cm}$. in diameter. Itching and redness persisted, the patient states, for two weeks after inoculation.

Another series of tests was made in confirmation of the first series, using the same solution as previously, using suprarenal tablets alone, and using cocain solution. At the same time a similar series was performed on my own forearm, the results of which were all negative. The results were absolutely confirmatory, similar reactions being produced as at the previous tests. An additional fact of interest at this time was that after the solutions had been applied about fifteen minutes a sudden motion of the patient's forearm spilled the solutions to the inner side of the forearm. Evident traces of the solution were found for many days over the areas which had been covered in the spilling. Severe itching and redness and distinct streaks were produced where the procain solution had traveled. The use of procain had been given up and the hands were practically clear save for slight scaliness in patches, and one or two dry fissures. No new lesions and no new vesicles had appeared.

A third series of tests was made on the forearm, using six solutions: (1) Ringer's solution; (2) procain E (Metz), 2 per cent., with epinephrin; (3) procain F (Metz), 2 per cent., with epinephrin; (4) procain $T$ (Hoechst), with epinephrin (different make); (5) cocain, 1 per cent., with epinephrin, and (6) sterile water. Within twenty-four hours there were redness and eleva- 
tion about 2,3 and 4 , greatest about 3 . There was the same intense itching, with redness extending for $5 \mathrm{~mm}$. about the region of inoculation, with slight elevation, the whole process, and the redness, lasting for nearly two weeks.

CASE 2.-Dr. B. has practiced dentistry for thirteen years and had used procain two years before symptoms appeared.

History.-In November, 1919, he began to have redness of the skin about the nails, with fissuring of the nails and adjacent skin and itching, especially of the left hand. Later a similar condition appeared between the fingers and on the palms. The left hand had always been most severely affected. The process had always been more localized about the fingers and nails than elsewhere. There had been itching and oozing, with much serum exuding at times. The nails had been rough, cracked, corrugated, with some suppuration, both at the base of the nails and below the nails at the finger tips. He had been using Whitfield's ointment part of the time, and had been careful in regard to the use of soap. For one period of three or four weeks, and for several shorter periods, he had been unable to work because of his hands.

Six days before his visit to my office in October, 1920, he was told by Dr. A. (referred to in the preceding case report), of the possibility that procain might be the causal agent. At that time he had fissures beneath the nails at the tips of the first and third fingers on the left hand, and about these two nails especially, together with severe general itching and burning of the hands. At that time the right hand had much the same feeling with areas of redness and oozing on the approximating surfaces of the third and fourth fingers. He gave up the use of procain on this date, and he stated that the itching and burning had become gradually less and that the hands had become drier and less red.

Examination.-On the day of the examination, six days after stopping the use of procain, the left hand showed considerable fissuring about the nails, with redness and exfoliation below the tips. The skin at the bases was thickened and rough as in a chronic paronychia. The first three fingers were dry, rough and scaly. The last two fingers were nearly normal. On the right hand the nails were much better than on the left, but were rough and more or less corrugated and dark colored. Between the third and fourth fingers there was an area of redness on the fourth finger, with slight vesiculation on the side toward the third finger. There was also a small, red, crusted area on the dorsum of the fourth finger just behind the nail where the ball of the third finger rested while working. His position at work, holding an instrument, showed closer approximation and pressure on the spots mentioned. On the right forearm, near the wrist, on the palmar side, was a small papule where procain had been dropped about five days previously. The patient also gave a story of accidentally squirting some of the solution from the needle on his face, near the nose, some time ago, followed in from twenty-four to forty-eight hours by intense itching and redness.

Course of Disease.--Five days after his first visit, his hands showed much improvement. The left hand showed practically all normal skin. There were no signs of the previous condition about the middle finger nail. The right hand was all clear except on the thumb side of the fourth finger where there were a few small papules, dull red in color, which were not itching. The area on the dorsum of the fourth finger had disappeared entirely. The patient stated that he must have washed his hands forty times the day before as a matter of experiment, but his hands seemed no worse. He was still continuing to use cocain for extraction. 
Skin Tests.-At this time skin tests were performed with (1) sterile water; (2) procain, 2 per cent.; (3) apothesin, 2 per cent.; (4) cocain, 2 per cent.; (5) a solution containing procain and epinephrin in Ringer's solution (solution four or five months old); (6) procain, 0.5 per cent. These solutions were applied and allowed to remain for thirty minutes. In twenty minutes a definite wheal, irregularly $5 \mathrm{~mm}$. in diameter, appeared about number 2 . About number 6 was a similar blanched area, but only $2 \mathrm{~mm}$. in diameter. About number 4 was a slightly red area with slight elevation. The other areas simply showed a redness at the point of rotation of the dental burr. In twenty-four hours there was a distinct red papule about number 2 and number 6 , with considerable itching. In forty-eight hours there was at number 2 a red papule, 5 or $6 \mathrm{~mm}$. in diameter. The redness was rather intense, and there had been much itching about this particular inoculation. There was a slight redness and elevation for an area $3 \mathrm{~mm}$. in diameter about number 5 . About number 6 there was a slightly larger area of redness with a little more elevation. All of these had itched to some extent but not as much as number 2 . The patient discontinued the use of procain and his hands have remained normal.

CASE 3.-Dr. C. has practiced dentistry for seventeen years and had used procain from two to three months before symptoms appeared.

History.-In August, 1919, Dr. C. began to have itching and burning of the hands, with considerable redness and the formation of blisters on the backs of the hands and between the fingers. At this time he had to give up work for three weeks on account of his hands. He had had urticaria occasionally but that is the only previous skin trouble which he had had. He began the use of "Novol," a procain preparation in Ringer's solution, about two months previously, in May or June, 1919. He dated the disturbance approximately to the time when he began to boil frequently this anesthetic solution for use. He believed that his hands became affected from the steam.

When he returned to work his hands were scaly, without any itching or redness. Within five days recurrence occurred, and he was away from his office for another period of three weeks. This time when he returned to his office his hands were in much better condition and practically normal. Three days after his return to work his hands became affected again, and at this time his face was much swollen and red, with considerable crusting. It was necessary for him to give up work for a period of three or four weeks. During the period when the disease was at its height the nails were discolored, brownish, cracked, and became separated from the bed, and later came out altogether. His hands were very greatly swollen, so that it was impossible to approximate the tips of the fingers. The area of involvement never extended above the edge of the coat sleeves and was only slight on the palms. The condition of both hands was about the same.

In the latter part of October he started to use rubber gloves all the time in his work and remained practically free from any skin disturbance of his hands. Since March, 1920, he has been wearing gloves in his practice only while using procain, and his hands have remained clear.

About three weeks before coming to my office he had not used his gloves for one day because his hands had been well so long. On this day he used procain on five or six patients, and two days later the second and third fingers of his left hand were much fissured, red, and oozing. He has returned to the use of gloves during the actual injection of procain, but he removes the gloves after injecting the anesthetic and putting away his instruments.

At the present time the first two fingers of the left hand are the only ones which show any variation from normal. Near the tips are red, slightly fissured 
scaly areas which burn and itch slightly. The rest of the hand is normal. During the extraction of teeth the first two fingers of the left hand are naturally within the mouth of the patient, while the right hand grasps the forceps. These two fingers are the ones which are involved, and it is fair to suppose that the condition on the two fingers of the left hand may be due to the procain oozing from the gums. He has never had procain injected for any purpose.

Skin Tests.-Skin tests were performed on the inner side of the left forearm with (1) sterile water; (2) a proprietary brand of procain; (3) procain (Metz), 2 per cent.; (4) procain, 1 per cent.; (5) cocain, 2 per cent, and (6) sterile water. He reported in twenty-four hours on the skin tests, stating that number 3 showed slight redness. In forty-eight hours examination revealed (1) no reaction; (2) an area of slight redness, about $5 \mathrm{~mm}$. in diameter, very slightly elevated; (3) a red area, 6 to $7 \mathrm{~mm}$. in diameter, redness most intense of all, with extension of redness in one direction for $1 \mathrm{~cm}$.; slightly more elevation than in 2 ; (4) an area about $5 \mathrm{~mm}$. in diameter, paler than 3 , with extension in one direction for about $1 \mathrm{~cm}$. The patient stated that redness and itching appeared on the day of examination; that he noticed nothing the day previous); (5) no reaction; (6) no reaction. The redness and slight itching persisted for ten days or two weeks.

SKIN TESTS

The skin tests in these cases were performed on the flexor surface of the forearm, the site of inoculation being produced by the twirling of a dental burr rather than by the use of a sharp-edged instrument. Sterile water was used as a control, and the various constituents of the procain solution and tablets were used in order to avoid the possibility of any interaction, and in order to test each substance separately. The reactions produced were observed for thirty minutes, after twentyfour hours, and again forty-eight hours after inoculation. A description of the results of these examinations has been given to aid in the interpretation.

The evidence offered by skin tests is debatable ground. In these three cases the evidence of these tests checks up with the clinical findings and is corroborated by the results of treatment. Further investigations are under way to find the number of persons sensitive to these various local anesthetics, and a report will be rendered at some later date. It is expecting too much to hope that the results will check as closely in all cases, and I feel that the final test, as Rackemann ${ }^{2}$ says, is the agreement between the skin tests and the patient's history before making definite conclusions from the evidence of the skin tests.

\section{SPECIFICITY OF REACTION}

There are certain points in these cases which will bear additional emphasis. In all three cases an eruption appeared on the face, in

2. Rackemann, F. M.: The Clinical Study of One Hundred and Fifty Cases of Bronchial Asthma, Arch. Int. Med. 22:517 (Oct.) 1918. 
all probability associated with the accidental application of procain. This fact, with the evidence of the skin tests, indicates the specificity of the reaction to this particular drug and that it is a systemic condition, not merely a localized disturbance. In one case there is the possibility of a concomitant infection with a fungus. In this relation it is possible to suppose that the moist, soggy condition of the skin produced by the dermatitis would act as an excellent medium for the growth of these organisms which may be almost normal inhabitants of the skin. In only one case was there evidence of immediate allergic reaction, as evidenced by the appearance of wheals at the sites of inoculation; but all three cases showed evidences of specific reaction by papules and redness at the points of inoculation forty-eight hours afterward.

Here are three persons apparently susceptible to a drug which they have handled extensively, who have shown signs of a dermatitis on areas which this drug has reached, who have reacted to skin tests specifically for this particular drug, and whose condition has cleared up on refraining from the use of this particular agent. Several other cases have come to my attention, though not seen by me personally, in which dentists with an irritated condition of the hands while using procain, have experienced much improvement in their condition after using rubber gloves constantly while handling such solutions. It would seem then that sufficient evidence is presented to affirm that a susceptibility to procain has been the cause of dermatitis in this group. Moreover, the lapse of time from the beginning of the use of the drug to the onset of the irritation suggests that the susceptibility is acquired rather than hereditary.

\section{ANAPHYLAXIS OR ALLERGY}

The fact of this susceptibility brings up for consideration the phenomena of anaphylaxis or allergy. It is an open question whether these persons have become sensitized by the constant handling of procain and whether this dermatitis is an allergic reaction. An attempt was made to ascertain whether it was possible that these persons had become sensitized by the previous use of procain internally, that is, at previous extractions of teeth or previous operations. No history of subcutaneous use of procain was obtained. Highman and Michael, ${ }^{3}$ in a recent paper, state that nothing is known of sensitization through normal integument, but that it is possible that the skin at these sites always presents microscopic injuries. In support of this they cite the case of an actor who developed angioneurotic edema following the application of grease paints, stating that this case may be a possible

3. Highman, W. J., and Michael, J. C.: Protein Sensitization in Skin Diseases: Urticaria and Its Allies, Arch. Dermat. \& Syph. 2:544 (Nov.) 1920. 
example of parenteral sensitization. They also cite cases of guineapigs sensitized by inhaling sprayed foreign serum, and also call attention to the fact that sensitization through injured integument is conceivable because of the fact that extremely small amounts of alien protein may sensitize.

Can this drug act as an alien protein? It is known that other substances than proteins are able to produce reactions similar to those above described. The eruptive phenomena occurring occasionally after the administration of arsphenamin have been sufficiently impressed on our minds. Auer ${ }^{4}$ states that the suggestion has been made that certain drug reactions may depend on the formation of compounds between the drug and the body proteins so that substances similar to foreign proteins are formed, and that this accounts for the resemblance to anaphylaxis which drug reactions may exhibit. This hypothesis, however, has not been established. In a summary of his experimental work along this line, he states that anaphylactic reaction may occur because the inflamed tissues are more active metabolically than normal tissues, and, therefore, the inflamed cells are affected by more antigen per unit of time than normal cells. Thus a lessened concentration of antigen for noninflamed sensitized cells may become effective when inflamed sensitized cells are concerned.

\section{CHEMICAL EXPLANATION OF ANALOGOUS CONDITION}

It is hardly possible at the present time to offer any explanation, even a partial one, on chemical grounds, but it is worth while to consider a somewhat analogous condition in rubber workers which has recently been described and explained on the basis of certain known chemical facts. The recent work of Shepard and Krall,5 while not explaining the underlying cause of susceptibility, still makes an ingenious explanation of the causes of dermatitis associated with certain rubber workers. Their explanation is based sufficiently on known facts to make it appear plausible, and further investigation of causes along similar lines is justified from the work which they have performed. They report that certain of the workers in a rubber factory, which uses hexamethylenamin as an accelerator in the vulcanizing process, developed a dermatitis on forearms, arms and face, and were found to have a susceptibility to hexamethylenamin. They offer an explanation of the inflammatory process based on the fact that hexamethylenamin

4. Auer, John: Local Auto-Inoculation of the Sensitized Organism with Foreign Protein as a Cause of Abnormal Reactions, J. Exper. Med. 32:427 (Oct.) 1920.

5. Shepard, N. A., and Krall, S.: Poisons in the Rubber Industry, The India Rubber World 61:75 (Nov. 1) 1919. 
is known to break down in the presence of acid and produce formaldehyd. The sweat is acid. The eruption is worse in hot weather. Formaldehyd alone does not produce a similar eruption. Formic acid does. They offer the explanation that possibly formaldehyd on the skin, in the hair follicles or glands, may be oxidized to formic acid, thus producing an irritation which is found in these workers. They offer as a solution to this condition the use of an alkaline solution. This treatment not only relieves the condition, but prevents its appearance, even in sensitized persons, when their hands and arms are immersed in the solution and allowed to dry before going to work. As I have said, no explanation is offered by these authors in regard to the underlying condition of susceptibility to hexamethylenamin, but the explanation of the cause of the dermatitis and its cure appears as a reasonable one.

\section{POSSIBILITY OF GENERALIZED REACTIONS}

It is interesting to conjecture as to the conditions which may be produced in persons susceptible to procain who subsequently receive injections for the extraction of teeth or for operative procedures. Do they have more local reaction, or do they have symptoms of a general nature? Dentists occasionally have patients who have become sick after a local anesthetic and may attribute that sickness to the excitement of the moment, to the actual extraction, possibly to sepsis afterward, or to the drug. There have not been, apparently, as many cases of illness produced after extraction of teeth since the use of procain, cocain apparently producing a greater effect on the person than the newer drugs. Three cases of death following procain have been found, one following extraction of teeth, and two following operative procedures on the antrum. All three of these were fairly sudden, and the case reports attribute these fatalities to the drug, although comment is made of the possibility that the operation and manipulation might have had some influence in the antrum cases.

The consideration of this subject of anaphylaxis in relation to dermatology by Highman and Michael, and Towle, ${ }^{6}$ in recent papers, gives an accurate estimate of the current opinion in regard to this subject. This subject is, as they say, wholly on a theoretical basis as yet, but the fact remains that more and more cases and more and more diseases are being considered as candidates for admission to this particular group of dermatoses, and further study and analysis of these and other conditions will undoubtedly contribute much toward their eventual explanation.

6. Towle, H. P.: Protein Sensitization in the Production of Skin Disease, Arch. Dermat. \& Syph. 2:531 (Nov.) 1920. 


\section{CONCLUSIONS}

1. In dermatitis of the hands in dentists procain must be considered as a possible causal factor.

2. The clinical observations, together with the confirmatory results from skin tests, indicate an individual susceptibility.

3. The possibility of sensitization by means of the skin in these cases is suggested.

4. The treatment is obvious - to prevent the drug from reaching the hands, either by supporting its use, or by wearing rubber gloves.

21 Bay State Road. 